\title{
Decentralized Trade, Random Utility and the Evolution of Social Welfare*
}

\author{
KANDORI, Michihiro ${ }^{\dagger}$ Roberto SERRANO ${ }^{\ddagger}$ and Oscar VOLIJ ${ }^{\S}$
}

July 12,2004

\begin{abstract}
We study decentralized trade processes in general exchange economies and house allocation problems with and without money. Such processes are subject to persistent random shocks stemming from agents' maximization of random utility. By imposing structure on the utility noise term - logit distribution-, one is able to calculate exactly the stationary distribution of the perturbed Markov process for any level of noise. We show that the stationary distribution places the largest probability on the maximizer of several social welfare functions in different variants of the model.

JEL classification numbers: C79, D51, D71.

Keywords: decentralized trade, exchange economies, housing markets, stochastic stability, logit model, social welfare functions.
\end{abstract}

\footnotetext{
${ }^{*}$ We thank Ryo Ogawa for implementing the simulations in Section 2.2. Serrano thanks the National Science Foundation and Deutsche Bank for research support, and the Institute for Advanced Study for its hospitality.

${ }^{\dagger}$ University of Tokyo, e-mail: kandori@e.u-tokyo.ac.jp

${ }^{\ddagger}$ Brown University, e-mail: roberto_serrano@brown.edu

${ }^{\S}$ Iowa State University and Ben-Gurion University of the Negev, e-mail: oscar@volij.co.il
} 


\section{Introduction}

This paper considers the allocation of indivisible durable goods through decentralized trading processes. A simple example is the allocation of $N$ offices among $N$ students. Even if the size of the problem, $N$, is relatively small, the number of possible allocations can be quite large. With ten students and offices, the number of allocations is about 3.6 million. When there are other goods to be allocated besides offices (such as parking permits), the problem of finding efficient allocations becomes even more complex. We examine how successful decentralized trading processes are in solving those complex combinatorial problems.

We consider a situation where agents randomly meet over time. When a group of agents meet, they exchange their goods in the following simple way. First, a new allocation for them is randomly proposed, and it is accepted if it provides a higher utility for all of them. Otherwise, the agents continue to hold their endowments. When they assess the proposed allocation, we assume that their utility is subject to random shocks. These shocks can be interpreted as mistakes, or temporal changes in tastes. Alternatively, the shocks may represent speculation based on "animal spirits" (that is, an agent may accept a bad bundle of goods for him, in the hope that it will be exchanged for a better bundle in the future; our "animal spirits" interpretation is that agents do not always hold rational expectations about the future course of exchange).

Incorporating random terms in utility function has been found to be quite useful in econometric studies of discrete choice problems (such as the choice of occupation or means of transportation), and we employ one of the leading specifications in econometrics, the logit model, for the distribution of the noise term. Owing to the special structure of the model, we obtain the closed form solution of the stationary distribution, for any level of noise. This is in contrast to the traditional stochastic stability methodology, first introduced to economics and game theory in Kandori, Mailath and Rob (1993) and Young (1993a). The method identifies those states -allocations- in which the economy spends most of its time in the long run, when the noise in the system is made negligible. Negligible noise implies a fairly long waiting time to see the long run effects, and this casts doubt on the relevance of the model. The present paper, in contrast, allows us to analyze the case where the noise level is reasonably large, so that the stationary distribution provides useful predictions over an economically relevant time horizon. It turns out that in our model, the selected state under vanishing noise remains the most likely state in the stationary distribution, for any level of noise. Specifically, we show that, for any level of noise, the state that maximizes a well known social welfare function receives the largest probability in the stationary distribution (and, as the randomness vanishes, the stationary distribution assigns probability one to such a state). We explore different variants of the model. For example, when the noise is additive, we find that the most likely state maximizes Bentham's utilitarian social welfare (the sum of utilities). Also, when the noise is multiplicative, the state visited most of the time is the one that maximizes Nash's social welfare (the product of utilities). If the trading process is implemented by (very) risk-averse representatives, the most likely state maximizes Rawls' egalitarian social welfare function (the minimum of utilities). All these results are a consequence of a single technical argument, relating stochastic stability with a strong form of utilitarianism.

Note that the above results imply that the most likely state is efficient. This conclusion may seem straightforward because (when the randomness is negligible) agents agree to trade only if their payoffs increase, so that the social welfare should (mostly) be monotone increasing. 
The important point to note, however, is that with no noise the process may be stuck on an inefficient state. For example, this will happen when only bilateral trades are possible once the economy reaches an inefficient state where there is no double coincidence of wants. ${ }^{1}$ In this respect, decentralized trading processes for indivisible goods resemble the algorithms that are used to solve combinatorial optimization problems with multiple local maxima, where the process may get stuck in one of them. For this, it has been found that random search algorithms, notably the ones based on simulated annealing methods (see Aarts and Korst (1989)) are quite effective. Since stochastic evolutionary game theory is based on the same basic idea as simulated annealing, its application to the allocation of indivisible goods should be particularly fruitful. Just like randomness in simulated annealing helps to escape from a local maximum, so does randomness in utility ensure that our trading process is not stuck at an inefficient state.

The above results are obtained for barter processes, but we also study exchange of goods with monetary transfers. It turns out that our assumption on the noise term and the quasi-linearity of utility in money allows us to extend the same techniques to this case, thereby yielding a similar result. This may be of independent interest: despite money being a continuous variable in the model, we are able to use the methods and framework developed mostly for discrete variables.

The paper is organized as follows. Section 2 analyzes three models of barter processes, and Section 3 introduces money. The final section discusses related literature.

\section{Decentralized Barter: Exchange Economies}

There are $K$ durable and indivisible commodities in the economy. The set of agents is $N=$ $\{1, \ldots, I\}$. Agent $i$ 's consumption set is $\left.Z_{i} \subset\{0,1,2, \ldots\}\right\}^{K}$. This allows for the possibility that an agent consumes an arbitrary number of units of each good, as in general exchange economies, or only one unit of one of the goods and zero of the others, as in house allocation problems. At time $t \in\{1,2, \ldots$,$\} agent i$ holds a bundle of commodities denoted by $z_{i}(t)$. Although the individuals' holdings may change over time, the aggregate endowment of goods remains fixed, i.e. $\sum_{i \in N} z_{i}(t)=\bar{z}$. A coalition is a non-empty subset of agents. For any coalition $S \subset N$, a feasible allocation for $S$ at time $t$, denoted by $z_{S} \in \times_{i \in S} Z_{i}$ is a distribution of their endowments at $t$. The set of feasible allocations for $S$ at $t$ is

$$
A_{S}\left(z_{S}(t)\right)=\left\{z_{S}^{\prime} \in \times_{i \in S} Z_{i} \mid \sum_{i \in S} z_{i}^{\prime}=\sum_{i \in S} z_{i}(t)\right\}
$$

The set of feasible allocations in the economy is given by

$$
Z=A_{N}(\bar{z})=\left\{z_{N} \in \times_{i \in N} Z_{i} \mid \sum_{i \in S} z_{i}^{\prime}=\bar{z}\right\}
$$

There is an exogenously given set of feasible coalitions, denoted $\mathbf{S} \subset 2^{N}$ that may meet and trade in each period. For example, when only pairwise meetings are possible (a particular case of

\footnotetext{
${ }^{1}$ Section 2.2 provides a simple example to illustrate this point. Ben-Shoham, Serrano, and Volij (2004) showed that an inefficient state can be stochastically stable, when all mistakes are equally likely. Hence, adding noise does not always help to escape from an inefficient state. Our model provides a set of sufficient conditions for the noise term to knock out inefficient states.
} 
our model), we have $\mathbf{S}=\{S \subset N|| S \mid=2\}$. We assume that from any initial feasible allocation $z$, any feasible allocation $z^{\prime}$ can be reached through a series of reallocations by a sequence of coalitions $S^{1}, \ldots, S^{T} \in \mathbf{S}$. At period $t=1,2, \ldots$ a coalition $S \in \mathbf{S}$ is selected with probability $q(S)>0$ (independently over time), and has the opportunity to reallocate their holdings of commodities.

\subsection{Barter with Additive Random Utility}

We introduce persistent random shocks by assuming that agent $i$ 's utility is given by

$$
v_{i}\left(z_{i}\right)=u_{i}\left(z_{i}\right)+\eta_{i}\left(z_{i}\right)
$$

where $u_{i}\left(z_{i}\right)$ and $\eta_{i}\left(z_{i}\right)$ stand for the intrinsic utility derived from bundle $z_{i}$ and noise, respectively. The noise term is independently distributed over time and across agents.

Suppose that, in the current period, a coalition $S \in \mathbf{S}$ is selected, and let $z_{S} \equiv z_{S}(t)$ be the allocation of goods for this coalition at the beginning of the current period. A new allocation for this coalition is determined as follows. There is a fixed probability distribution, which we call the proposal distribution, over the set of new feasible allocations $A_{S}\left(z_{S}(t)\right)$. We assume that this distribution is uniform: a new allocation $z_{S}^{\prime} \in A_{S}\left(z_{S}(t)\right)$ is proposed with probability $1 /\left|A_{S}\left(z_{S}(t)\right)\right|$. When coalition $S$ meets and is considering a proposal $z_{S}^{\prime}$, alternative to the current $z_{S}$, we assume that the new allocation is implemented if and only if $\forall i \in S, v_{i}\left(z_{i}^{\prime}\right) \geq v_{i}\left(z_{i}\right)$, with a strict inequality at least for one agent.

Therefore, if we let $F_{i}$ be the distribution of the noise term $\eta_{i}\left(z_{i}\right)$ for all $z_{i}$, for any two bundles $z_{i}$ and $z_{i}^{\prime}$, the probability that agent $i$ agrees to get $z_{i}^{\prime}$ in exchange for $z_{i}$ is given by

$$
\begin{aligned}
\operatorname{Pr}\left(v_{i}\left(z_{i}^{\prime}\right)>v_{i}\left(z_{i}\right)\right) & =\operatorname{Pr}\left(u_{i}\left(z_{i}^{\prime}\right)-u_{i}\left(z_{i}\right)>\eta_{i}\left(z_{i}\right)-\eta_{i}\left(z_{i}^{\prime}\right)\right) \\
& =\int_{-\infty}^{\infty} F_{i}\left(u_{i}\left(z_{i}^{\prime}\right)-u_{i}\left(z_{i}\right)+x\right) d F_{i}(x) .
\end{aligned}
$$

Assume further that the noise term $\eta_{i}\left(z_{i}\right)$ in (1) has the type I extreme value distribution (or Gumbel distribution) with precision parameter $\beta_{i}>0$, whose cumulative distribution function $F_{i}$ is given by

$$
F_{i}(x)=\exp \left(-\exp \left(-\beta_{i} x-\gamma\right)\right)
$$

where $\gamma$ is a constant to make the mean equal to zero. Then it is known that

$$
\operatorname{Pr}\left(v_{i}\left(z_{i}^{\prime}\right)>v_{i}\left(z_{i}\right)\right)=\frac{\exp \left[\beta_{i} u_{i}\left(z_{i}^{\prime}\right)\right]}{\exp \left[\beta_{i} u_{i}\left(z_{i}^{\prime}\right)\right]+\exp \left[\beta_{i} u_{i}\left(z_{i}\right)\right]} .
$$

From this formula it can be seen that, as $\beta_{i} \rightarrow \infty$, noise vanishes and the agent maximizes $u_{i}$ without any error. That is,

$$
\lim _{\beta_{i} \rightarrow \infty} \operatorname{Pr}\left(v_{i}\left(z_{i}^{\prime}\right)>v_{i}\left(z_{i}\right)\right)= \begin{cases}1 & \text { if } u_{i}\left(z_{i}^{\prime}\right)>u_{i}\left(z_{i}\right) \\ 0 & \text { if } u_{i}\left(z_{i}^{\prime}\right)<u_{i}\left(z_{i}\right)\end{cases}
$$

This is what is known in econometrics as the logit model. As is clear from the first equality in (2), what matters for the calculation of the probability that agent $i$ accepts to trade is the distribution of $t=\eta_{i}\left(z_{i}\right)-\eta_{i}\left(z_{i}^{\prime}\right)$. Under our assumption on $F_{i}$, this distribution is given by $G_{i}(t)=1 /\left(1+e^{-\beta_{i} t}\right)$, whose density function is $g_{i}(t)=\beta_{i} e^{-\beta_{i} t} /\left(1+e^{-\beta_{i} t}\right)^{2}$. This density function, for $\beta_{i}=1$, is plotted in Figure 1. 


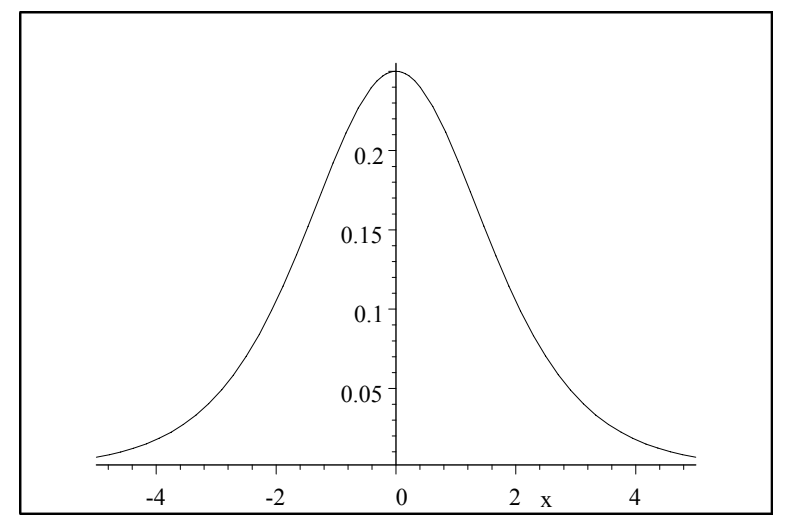

Figure 1.

As we can see, the density is quite similar to the normal density, but it has fatter tails. ${ }^{2}$ Although the normal noise has better "microfoundations" (as the sum of independent shocks), it does not allow a clean analytical solution. Instead, we use the logit model, which is similar to the normal one, but which does provide a nice closed form solution. Later, in Section 2.2, we conduct numerical simulations with normal noise to show that the behavior of both models is quite similar to each other.

The above description defines a Markov process on the set of feasible allocations of the economy. At every period, the economy can transit from one allocation to another and, since we assumed that the set of feasible coalitions is such that it is always possible to go from any feasible allocation to any other through a finite sequence of reallocations, the resulting Markov process is irreducible. Moreover, there is a chance that the state does not change, which indicates that the process is aperiodic. For such a process, there is a unique stationary distribution with the following two properties. First, starting from any initial allocation, the probability distribution on period $t$ allocations is known to approach that stationary distribution (as $t \rightarrow \infty)$. Second, the stationary distribution also represents the proportion of time spent on each state over an infinite time horizon.

Proposition 1 In the barter model with additive random utility, the stationary distribution is given by

$$
\mu(z)=\frac{\exp \sum_{i \in N} \beta_{i} u_{i}\left(z_{i}\right)}{\sum_{z^{\prime} \in Z} \exp \sum_{i \in N} \beta_{i} u_{i}\left(z_{i}^{\prime}\right)} .
$$

Before we present the proof, a few remarks are in order. First, the denominator is a normalizing constant, common to all $z$, to ensure that $\sum_{z \in Z} \mu(z)=1$. Therefore only the numerator contains relevant information. The formula tells us that the stationary distribution is "exponentially

\footnotetext{
${ }^{2}$ The tail of the normal density decays at the rate of $e^{-c t^{2}}$, while the density $g_{i}(t)$ for the logit case approximately decays at the rate of $e^{-c^{\prime} t}$ for large $t$ (see the expression for $g_{i}(t)$ : the constant $c^{\prime}$ is equal to the precision parameter $\beta_{i}$.). As $t \rightarrow \infty$, the former converges to zero faster than the latter. (Of course, $c=1 / 2 \sigma^{2}$, where $\sigma^{2}$ is the variance of the normal distribution. Since the variance of the logit noise difference $g_{i}(t)$ is known to be $\pi^{2} / 3 \beta_{i}^{2}$, for more precise comparisons we could set $\sigma^{2}=\pi^{2} / 3 \beta_{i}^{2}$. We will conduct such comparisons in Section 2.2.)
} 
proportional" to the social welfare function $\sum_{i \in N} \beta_{i} u_{i}\left(z_{i}\right)$. In particular, the most likely state (for any level of noise) is the one that maximizes that social welfare. Second, recall that $\beta_{i}$ is the precision parameter of agent $i$ 's noise term, meaning that a larger $\beta_{i}$ implies a smaller level of noise. The formula is easiest to understand when we regard the noise term as the representation of mistakes. Suppose two players have identical utility functions but different precision paramenters. Then, the agent who makes fewer mistakes (i.e., who had the higher $\beta_{i}$ ) receives a higher utility in the long run. Third, the stationary distribution is independent of the matching probabilities, represented by $q(s)$. Suppose that we have two players with identical utility functions and precision parameters, and assume that one has more opportunities to trade than the other. One may expect that the one with more opportunities to trade does better than the other, but in the long run they receive the same payoff distribution.

Proof. Let $\operatorname{Pr}\left(z, z^{\prime}\right)$ be the transition probability from state $z$ to $z^{\prime}$. It is enough to show that

$$
\mu(z) \operatorname{Pr}\left(z, z^{\prime}\right)=\mu\left(z^{\prime}\right) \operatorname{Pr}\left(z^{\prime}, z\right) \quad \forall z, z^{\prime} \in Z .
$$

To see that this is sufficient, note that by summing both sides over all $z^{\prime} \in Z$ we get

$$
\mu(z)=\sum_{z^{\prime} \in Z} \mu\left(z^{\prime}\right) \operatorname{Pr}\left(z^{\prime}, z\right) \quad \forall z \in Z,
$$

which means that $\mu$ is an invariant distribution. Equation (6), a sufficient condition for stationary distribution, is known as the detailed balance condition, and it states that the probability inflows and outflows are balanced for any pair of states. To show that the detailed balance condition holds, it suffices to show that

$$
\left(\exp \sum_{i \in N} \beta_{i} u_{i}\left(z_{i}\right)\right) \operatorname{Pr}\left(z, z^{\prime}\right)=\left(\exp \sum_{i \in N} \beta_{i} u_{i}\left(z_{i}^{\prime}\right)\right) \operatorname{Pr}\left(z^{\prime}, z\right) .
$$

Let $S^{\prime} \equiv\left\{i \in N \mid z_{i}^{\prime} \neq z_{i}\right\}$ be the set of agents whose bundles change, and let $\mathbf{S}^{\prime} \equiv\left\{S \in \mathbf{S} \mid S^{\prime} \subset S\right\}$ be the set of feasible coalitions containing $S^{\prime}$. The new allocation $z^{\prime}$ is obtained from $z$ only if a coalition in $\mathbf{S}^{\prime}$ is selected. If $\mathbf{S}^{\prime}$ is empty, we have $\operatorname{Pr}\left(z, z^{\prime}\right)=\operatorname{Pr}\left(z^{\prime}, z\right)=0$ so that the condition (6) is satisfied. Otherwise, we have

$$
\operatorname{Pr}\left(z, z^{\prime}\right)=\sum_{S \in \mathbf{S}^{\prime}} q(S) \frac{1}{\left|A_{S}\left(z_{S}\right)\right|} \frac{\exp \left[\sum_{i \in S^{\prime}} \beta_{i} u_{i}\left(z_{i}^{\prime}\right)\right]}{H},
$$

where $H=\prod_{i \in S^{\prime}}\left\{\exp \left[\beta_{i} u_{i}\left(z_{i}^{\prime}\right)\right]+\exp \left[\beta_{i} u_{i}\left(z_{i}\right)\right]\right\}$. Note that $q(S)$ is the probability that coalition $S$, which contains $S^{\prime} \equiv\left\{i \in N \mid z_{i}^{\prime} \neq z_{i}\right\}$, is selected, and with probability $\frac{1}{\left|A_{S}\left(z_{S}\right)\right|}$ the new allocation $z_{S}^{\prime}$ is proposed (recall our uniform proposal assumption). The third factor is, by (5), the probability that the agents who are proposed a new allocation (i.e., agents in $S^{\prime} \equiv\left\{i \in N \mid z_{i}^{\prime} \neq z_{i}\right\}$ ) accept the proposal. ${ }^{3}$

Similarly, we have

$$
\operatorname{Pr}\left(z^{\prime}, z\right)=\sum_{S \in \mathbf{S}^{\prime}} q(S) \frac{1}{\left|A_{S}\left(z_{S}^{\prime}\right)\right|} \frac{\exp \left[\sum_{i \in S^{\prime}} \beta_{i} u_{i}\left(z_{i}\right)\right]}{H} .
$$

\footnotetext{
${ }^{3}$ Agents in $S \backslash S^{\prime}$ are proposed the same bundles as before, so they are indifferent between $z_{S}^{\prime}$ and $z_{S}$.
} 
Hence, if we denote $U(z) \equiv \sum_{i \in S^{\prime}} \beta_{i} u_{i}\left(z_{i}\right)$, we have

$$
\begin{aligned}
(\exp U(z)) \operatorname{Pr}\left(z, z^{\prime}\right) & =\sum_{S \in \mathbf{S}^{\prime}} q(S) \frac{1}{\left|A_{S}\left(z_{S}\right)\right|} \frac{\exp \left[\left\{U(z)+\sum_{i \in S^{\prime}} \beta_{i} u_{i}\left(z_{i}^{\prime}\right)\right\}\right]}{H} \\
& =\sum_{S \in \mathbf{S}^{\prime}} q(S) \frac{1}{\left|A_{S}\left(z_{S}\right)\right|} \frac{\exp \left[\left\{U\left(z_{-S}, z_{S}^{\prime}\right)+\sum_{i \in S} \beta_{i} u_{i}\left(z_{i}\right)\right\}\right]}{H} \\
& =\sum_{S \in \mathbf{S}^{\prime}} q(S) \frac{1}{\left|A_{S}\left(z_{S}^{\prime}\right)\right|} \frac{\exp \left[\left\{U\left(z^{\prime}\right)+\sum_{i \in S^{\prime}} \beta_{i} u_{i}\left(z_{i}\right)\right\}\right]}{H} \\
& =\exp U\left(z^{\prime}\right) \operatorname{Pr}\left(z^{\prime}, z\right),
\end{aligned}
$$

where we used the fact that $A_{S}\left(z_{S}\right)=A_{S}\left(z_{S}^{\prime}\right)$, because $\sum_{i \in S} z_{i}=\sum_{i \in S} z_{i}^{\prime}$. Hence condition (7) is satisfied

Note that the detailed balance equation (6) fails when the proposal distribution is not uniform over the allocations other than the current one. Without the uniform assumption, the clean closed form solution cannot be obtained. On the other hand, in house allocation problems, where each agent occupies one house, this assumption is automatically satisfied when only pairwise meetings are possible. This is because the only feasible new allocation for a pair is obtained by just exchanging their houses.

Thus, Proposition 1 allows one to obtain the exact proportion of time that the system would spend at each feasible allocation in the long run. Let us now examine how the stationary distribution changes with the level of noise. For simplicity, consider the symmetric case with $\beta_{1}=\cdots=\beta_{I}=\beta$. When the precision parameter of the noise terms $\beta$ is close to 0 , the system is subject to large random shocks, and the expression in Proposition 1 shows that the stationary distribution is close to the uniform distribution. As the level of noise decreases (i.e., as $\beta$ increases), states with higher social welfare $\sum_{i \in N} u_{i}\left(z_{i}\right)$ receive higher probabilities. When noise is vanishing $(\beta \rightarrow \infty)$, each term $\exp \beta \sum_{i \in N} u_{i}\left(z_{i}\right), z \in Z$ diverges to infinity, but the one that corresponds to the maximizer of the social welfare $\sum_{i \in N} u_{i}\left(z_{i}\right)$ does so with the highest speed. Hence we have the following sharp characterizations.

Corollary 2 In the barter model with additive random utility, if the noise is symmetric $\left(\beta_{1}=\right.$ $\cdots=\beta_{I}=\beta$ ) and $\beta \rightarrow \infty$, the stationary distribution places probability 1 on the allocation(s) that maximizes the Bentham utilitarian social welfare function $\sum_{i \in N} u_{i}\left(z_{i}\right)$.

Corollary 3 In the barter model with additive random utility, if the noise parameter is $\beta_{i}=\lambda_{i} \beta$ for all $i \in N$ and $\beta \rightarrow \infty$, the stationary distribution places probability 1 on the allocation(s) that maximizes the corresponding weighted utilitarian social welfare function $\sum_{i \in N} \lambda_{i} u_{i}\left(z_{i}\right)$.

\section{$2.2 \quad$ Numerical Examples}

The stationary distribution we have obtained summarizes the behavior of the system over a long horizon. To investigate the behavior of the sample paths in more detail, we conduct some numerical simulations. We also examine if the normal noise case, which does not admit an analytical solution, behaves in a similar way to our logit model. 
Suppose that we have 30 students and 30 parking permits. The parking permits come in three varieties. There are 10 type $a$ permits, which allow one to park in area $a$. Similarly, we have 10 type $b$ and 10 type $c$ permits. There are also three types of students, with the following utility functions:

$$
\begin{aligned}
& \text { Type } A \quad: \quad u_{A}(a)=2, \quad u_{A}(b)=1, \quad u_{A}(c)=0 \\
& \text { Type } B \quad: \quad u_{B}(b)=2, \quad u_{A}(c)=1, u_{A}(a)=0 \\
& \text { Type } C: \quad u_{A}(c)=2, u_{A}(a)=1, u_{A}(b)=0
\end{aligned}
$$

That is, the most convenient parking areas for type $A, B$, and $C$, are $a, b$, and $c$ respectively. There are 10 students of each type. The following is the initial allocation, where no one has the most convenient permit.

$\begin{array}{llll} & a & b & c \\ A & 0 & 5 & 5 \\ B & 5 & 0 & 5 \\ C & 5 & 5 & 0\end{array}$

This means that, out of the 10 type $A$ players, five have permits $b$ and the other five have permits $c$. We assume pairwise meetings, so that one pair of students is randomly selected in each period and, if they agree, they exchange their permits. We first assume that each agent's noise term has type I extreme value distribution (the logit model) with the same precision parameter $\beta$. Figure 2 plots a typical sample path for our model. To summarize the data in one figure, we plot the total social welfare $\sum_{i \in N} u_{i}\left(z_{i}\right)$ (i.e., the sum of intrinsic utilities without noise).

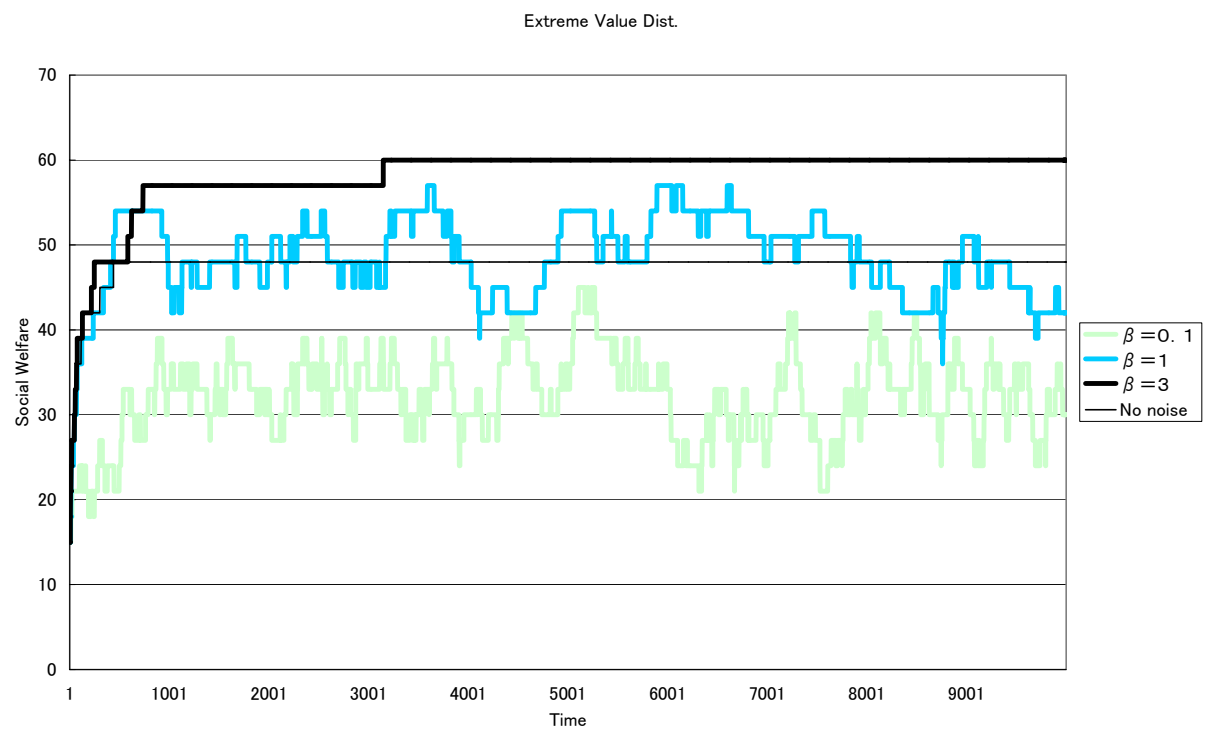

Figure 2.

Note that the maximum social welfare $\sum_{i \in N} u_{i}\left(z_{i}\right)$ is 60 (each of the 30 students receives maximum payoff 2). The figure shows that the process without noise can be stuck in an inefficient state. 
This occurs when each student ends up having his second best permit, so that there is no double coincidence of wants. For example, assume that type $A$ students have permit $b$ and type $B$ students have permit $c$. When they meet, $B$ students would like to obtain their best permit $b$, while $c$ is the worst one for $A$ students. Hence (without noise) they do not agree to trade.

When the level of noise is large $(\beta=0.1)$, the process is volatile and the sum of intrinsic utilities is generally low. However, for lower randomness $(\beta=1)$, the social welfare increases, and when the level of noise is reasonably small $(\beta=3)$, the process reaches the maximum social welfare and stays there for a long time. The variance of the noise term $\eta_{i}\left(z_{i}\right)$ in this case is $\frac{\pi^{2}}{6 \beta^{2}} \approx$ 0.18. If we assume that three pairs meet per day, the figure shows that the maximum social welfare is achieved within three years. ${ }^{4}$

We now present a typical simulation result with normally distributed noise terms (Figure 3). The path for $\beta=1$ in the figure, for example, is associated with the normal noise which has the same variance as the type I extreme value distribution with $\beta=1$.

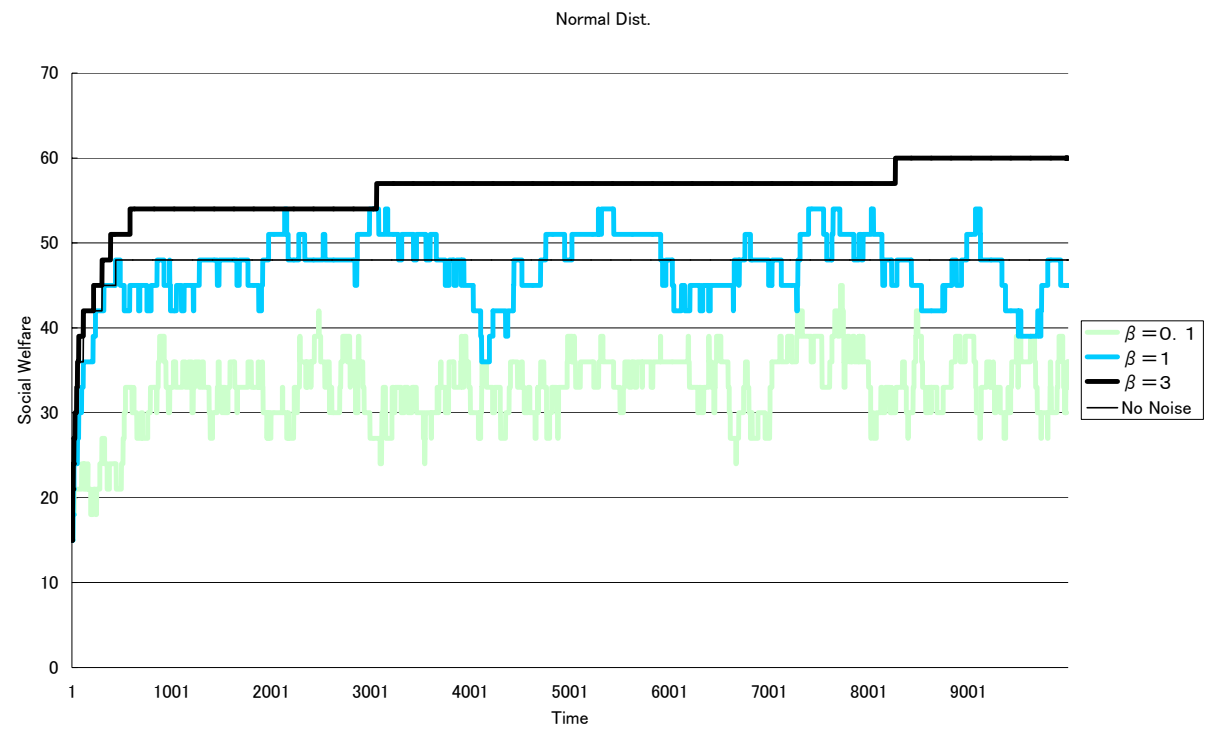

Figure 3.

We can see that the behavior of the paths are similar to that of the logit model, although convergence to the maximum social welfare is slower in the case where $\beta=3$. This may be due to the fact that the type I extreme value distribution has fatter tails than the normal distribution, so that a large disturbance, which helps to leave an inefficient state, is less likely to occur under the normal distribution.

\footnotetext{
${ }^{4}$ The term "social welfare" should be interpreted carefully. If the noise term represents mistakes and animal spirit speculation (see introduction), then the interpretation is straightforward. In contrast, if the noise term represents payoff shocks, the realized payoffs (cum noise) is the relevant measure of social welfare. In that case, we cannot readily say that the path for $\beta=0.1$ is worse than the path without noise. However, this is not a problem when we compare the path without noise with the path for $\beta=3$. Note that those paths are mostly constant and that the latter dominates the former. Because the i.i.d. noise term has mean 0 , the (time) average of realized social welfare (cum noise) should be higher on the latter path.
} 


\subsection{Barter with Multiplicative Random Utility}

Now assume that the noise term in the utility function has a multiplicative form

$$
v_{i}\left(z_{i}\right)=u_{i}\left(z_{i}\right) \xi_{i}\left(z_{i}\right)
$$

where $\eta_{i}\left(z_{i}\right) \equiv \log \xi_{i}\left(z_{i}\right)$ has an extreme value distribution with parameter $\beta_{i}$, whose cumulative distribution function is given by (4). Then we have

$$
\log v_{i}\left(z_{i}\right)=\log u_{i}\left(z_{i}\right)+\eta_{i}\left(z_{i}\right)
$$

and exactly the same analysis as above applies, where $u_{i}\left(z_{i}\right)$ is now replaced with $\log u_{i}\left(z_{i}\right)$. Hence we have the following:

Proposition 4 In the barter model with multiplicative random utility, the stationary distribution is given by

$$
\mu(z)=\frac{\prod_{i \in N} u_{i}\left(z_{i}\right)^{\beta_{i}}}{\sum_{z^{\prime} \in Z} \prod_{i \in N} u_{i}\left(z_{i}^{\prime}\right)^{\beta_{i}}} .
$$

Using this closed form solution of the stationary distribution, one can obtain the following limiting results:

Corollary 5 In the barter model with multiplicative random utility, if the noise is symmetric $\left(\beta_{1}=\cdots=\beta_{I}=\beta\right)$ and $\beta \rightarrow \infty$, the stationary distribution places probability 1 on the allocations that maximize the Nash social welfare function $\prod_{i \in N} u_{i}\left(z_{i}\right)$.

Corollary 6 In the barter model with multiplicative random utility, if the noise parameter is $\beta_{i}=\lambda_{i} \beta$ for all $i \in N$ and $\beta \rightarrow \infty$, the stationary distribution places probability 1 on the allocation that maximize the weighted Nash social welfare function $\prod_{i \in N} u_{i}\left(z_{i}\right)^{\lambda_{i}}$.

For the Nash social welfare function, the reader is referred, for example, to Kaneko (1980). As for its weighted version, other names that have been used to describe this function are "generalized Nash solution" (Harsanyi and Selten (1972), p. 101) or "asymmetric Nash solution" (Kalai (1977)). (Roth ((1979), pp.15-16) provides a good exposition.)

The utility in the multiplicative case is the $\log$ of the utility in the additive case. One could argue that they both represent the same underlying (random) preference relations. The separate treatment of those two cases is justified when one admits that the utility has some cardinal meaning. For example, if we postulate that the intrinsic utility function $u_{i}$ is a von NeumannMorgenstern utility function, the multiplicative case is different from the additive case, because they represent different attitudes toward risk.

As an illustration of the economic relevance of both models, suppose a planner knows that half of the agents in a population are risk neutral (say, agents of type 2), while half have a square root utility (type 1) over some discrete units of money. In fact, to simplify let us suppose there are only two individuals who keep bumping into each other and reallocating a discrete surplus of size 1. In these meetings, accepting a reallocation of the pie is driven by the noise, which makes each bargainer "soft" and inclined to accept sometimes reductions of his share. The planner 
knows that the intrinsic utilities are as described, but is not sure which specification of logit noise accompanies intrinsic utility. At the same time, she knows that agents really are maximizing their intrinsic utility affected by noise. If noise is additive, the system will gravitate most of the time around the $(1 / 4,3 / 4)$ split of the discrete pie when mistakes are very unlikely, or around this and nearby splits that are in the direction of maximizing the sum of intrinsic utilities when noise is sizable (this split corresponds to the Bentham optimum). In contrast, if noise is multiplicative, it will tend to be around $(1 / 3,2 / 3)$ most of the time (the Nash optimum). That is, the additive noise specification punishes the risk averse agent more than does the multiplicative noise specification.

Young (1993b) obtains asymmetric Nash solutions as the limits of an evolutionary process in which pairs of agents play the Nash demand game. The process is one of perturbed best-response dynamics to a sample of past history. There are two populations of agents from which a pair of bargainers is drawn every period, and as a function of the sample size of each group, the system selects the corresponding asymmetric Nash solution, where the weights are given by the relative sample sizes.

\subsection{A Model of Representation}

We turn now to a conceptual variant of the model presented above. Suppose there is a set $N=\{1,2, \ldots, I\}$ of social groups. Group $i \in N$ has a (non-random) quasi-linear utility function $u_{i}\left(z_{i}\right)+t_{i}$, where $t_{i}$ represents the amount of money, and $z_{i}$ is the allocation of durable goods for group $i$. The group utility function $u_{i}\left(z_{i}\right)$ admits a couple of interpretations. We could interpret $u_{i}$ as the utility of the "representative" agent in group $i$. For more precise formulation, let $i_{1}, \ldots$, $i_{M}$ be the agents in group $i$, and assume that each agent $i_{m}$ has a quasi-linear utility $w_{i_{m}}(\cdot)+t_{i_{m}}$. When $z_{i}$ represents (local) public goods, the group utility function is given by $u_{i}\left(z_{i}\right)=\sum_{m} w_{i_{m}}\left(z_{i}\right)$. If $z_{i}$ consists of private goods, we have $u_{i}\left(z_{i}\right)=\max \sum_{m} w_{i_{m}}\left(z_{i_{m}}\right)$ s.t. $\sum_{m} z_{i_{m}}=z_{i}$. (Here we assume that the agents in the group optimally allocate $z_{i}$ among themselves (without mistakes).) In any case, note that $u_{i}\left(z_{i}\right)$ represents the dollar value of group $i$ 's possessions $z_{i}$. Each group hires a representative who is responsible for trading goods with the representatives of the other groups. In particular, when the representative of group $i$ obtains a bundle of goods $z_{i}$, he receives a share of the dollar-value of the bundle, $\theta u_{i}\left(z_{i}\right)$. For ease of analysis, we normalize the parameters so that $\theta=1$. We assume that the representative of group $i$ has a constant relative risk aversion utility function so that his intrinsic utility is given by $\frac{u_{i}\left(z_{i}\right)^{1-\rho_{i}}}{1-\rho_{i}}$, where $\rho_{i} \geq 0$ is his rate of relative risk aversion. In the trading process his utility is subject to random shocks, and he maximizes

$$
v_{j}\left(z_{i}\right)=\frac{u_{i}\left(z_{i}\right)^{1-\rho_{i}}}{1-\rho_{i}}+\eta_{i}\left(z_{i}\right)
$$

where $\eta_{i}$ has an extreme value distribution with precision parameter $\beta$. With the same proof as the first proposition, one can establish the following result:

Proposition 7 In the barter model with representation, the stationary distribution is given by

$$
\mu(z)=\frac{\exp \beta \sum_{i \in N} u_{i}\left(z_{i}\right)^{1-\rho_{i}} /\left(1-\rho_{i}\right)}{\sum_{z^{\prime} \in Z} \exp \beta \sum_{i \in N} u_{i}\left(z_{i}^{\prime}\right)^{1-\rho_{i} /\left(1-\rho_{i}\right)}} .
$$


Now, taking the limit as $\beta \rightarrow \infty$, we obtain another utilitarian selection because the process ends up assigning all probability to the allocations that maximize the sum of the utilities of the representatives. In particular,

Corollary 8 In the barter model with representation, if the relative risk aversion of each representative is the same $\left(\rho_{i}=\rho\right.$ for every representative $\left.i\right)$, as $\beta \rightarrow \infty$, the stationary distribution places probability 1 on the allocations that maximize the "constant elasticity of substitution" social welfare function $\sum_{i \in N} u_{i}\left(z_{i}\right)^{1-\rho} /(1-\rho)$. Moreover, there is $\bar{\rho}$ such that if $\rho>\bar{\rho}$, as $\beta \rightarrow \infty$, the stationary distribution places probability 1 on the allocations that maximize the "Rawlsian" social welfare function $\min _{i \in N}\left\{u_{i}\left(z_{i}\right)\right\}$.

According to this result, the more risk averse the group representatives are, the more egalitarianlike the social outcome is. That is, if $\rho=0$, which corresponds to representatives' risk neutrality, the outcome will be the maximizer of the utilitarian social welfare. However, as $\rho \rightarrow \infty$, the allocations selected with probability 1 are those for which the egalitarian Leontieff (or Rawlsian) social welfare function $\min _{i \in N}\left\{u_{i}\left(z_{i}\right)\right\}$ is maximized. (Our claim that this happens for a finite (but large) $\rho$ follows from the finiteness of feasible allocations: when $\sum_{i \in N} u_{i}\left(z_{i}\right)^{1-\rho} /(1-\rho)$ is sufficiently close to its limit $\min _{i \in N}\left\{u_{i}\left(z_{i}\right)\right\}$, they are maximized at the same point, because the domain of those functions is finite.) This may raise an intriguing conjecture for political theory: are egalitarian policies driven by risk averse politicians?

\section{Trade with Money: House Allocation Problems with Side Pay- ments}

We now consider the case where indivisible goods are traded with (divisible) money. While the barter model of the previous section may be a good approximation of the office allocation in a department where no monetary transfers are associated with the office assignment, to describe a housing market it is realistic to introduce monetary transfers.

More specifically, we consider an economy with a set $H$ of houses, and a set $N$ of agents. The number of houses and the number of agents are the same: $|H|=|N|$. An agent's consumption bundle consists of only one house and money. Therefore, a house allocation is an assignment $\left(z_{i}\right)_{i \in N}$ of the houses in $H$ to the agents in $N$. A typical allocation is an object of the form $\left(\left(z_{i}, m_{i}\right)\right)_{i \in N}$ such that $\left(z_{i}\right)_{i \in N}$ is a house allocation, and for each $i \in N, m_{i}$ denotes agent $i$ 's money holdings, which for simplicity are allowed to be negative.

Each agent $i \in N$ is assumed to have quasi-linear utility:

$$
\pi_{i}\left(z_{i}, m_{i}\right) \equiv v_{i}\left(z_{i}\right)+m_{i}=u_{i}\left(z_{i}\right)+\eta_{i}\left(z_{i}\right)+m_{i} .
$$

As before, $\eta_{i}\left(z_{i}\right)$ is the random component of utility and it is distributed according to the type I extreme value distribution with precision parameter $\beta_{i}$. Here we assume that the agents have the same parameter: $\beta_{i}=\beta$ for all $i \in N$. This turns out to be essential for the present analysis.

For now we only consider bilateral meetings in which a pair of agents $(i, j)$ is selected with probability $q(i, j)>0$ in each period. At the end of the section we shall discuss the extension of our analysis to general exchange economies with money and to a trading process involving 
coalitions other than pairs of agents. We consider the following bargaining procedure. Suppose agents $i$ and $j$ meet, with the current endowments $z_{i}$ and $z_{j}$. Let $p \in \Re$ be a monetary transfer from $i$ to $j$, where $i<j$. In other words, we follow a convention that $p$ denotes the payment made by the agent with a lower index, and note that this is without loss of generality, as $p$ can be negative. We suppose that the matched pair first come up with $p$ randomly, and then choose to trade at that price if this is mutually beneficial (according to their utilities with realized noise term). More specifically, let $f_{i j}(p)$ be the density of $p$ for pair $(i, j)$. Its support may be a finite interval which may vary across different pairs. We assume that this distribution is symmetric: $f_{i j}(p)=f_{i j}(-p)$. When $i$ and $j$ meet, first $p$ is realized according to $f_{i j}(p)$, and then they exchange their current holdings at price $p$ if and only if

$$
\begin{gathered}
u_{i}\left(z_{j}\right)+\eta_{i}\left(z_{j}\right)-p>u_{i}\left(z_{i}\right)+\eta_{i}\left(z_{i}\right), \text { and } \\
u_{j}\left(z_{i}\right)+\eta_{j}\left(z_{i}\right)+p>u_{j}\left(z_{j}\right)+\eta_{j}\left(z_{j}\right) .
\end{gathered}
$$

Then, as the random utility shocks $\eta_{i}(\cdot)$ and $\eta_{j}(\cdot)$ have an extreme value distribution, condition (11) holds with probability

$$
\frac{\exp \left(\beta\left(u_{i}\left(z_{j}\right)-p\right)\right)}{\exp \left(\beta\left(u_{i}\left(z_{j}\right)-p\right)\right)+\exp \left(\beta u_{i}\left(z_{i}\right)\right)} .
$$

Similarly, condition (12) holds with probability

$$
\frac{\exp \left(\beta\left(u_{j}\left(z_{i}\right)+p\right)\right)}{\exp \left(\beta\left(u_{j}\left(z_{i}\right)+p\right)\right)+\exp \left(\beta u_{j}\left(z_{j}\right)\right)} .
$$

Hence, given $p$, trade occurs with the product of the above probabilities, which is equal to

$$
\frac{\exp \left[\beta\left(u_{i}\left(z_{j}\right)+u_{j}\left(z_{i}\right)\right)\right]}{H(p)}
$$

where

$$
\begin{aligned}
H(p)= & {\left[\exp \left(\beta\left(u_{i}\left(z_{j}\right)-p\right)\right)+\exp \left(\beta\left(u_{i}\left(z_{i}\right)\right)\right]\left[\exp \left(\beta\left(u_{j}\left(z_{i}\right)+p\right)\right)+\exp \left(\beta\left(u_{j}\left(z_{j}\right)\right)\right]\right.\right.} \\
= & \exp \left(\beta\left(u_{i}\left(z_{j}\right)+u_{j}\left(z_{i}\right)\right)\right)+\exp \left(\beta\left(u_{i}\left(z_{j}\right)+u_{j}\left(z_{j}\right)-p\right)\right. \\
& +\exp \left(\beta\left(u_{i}\left(z_{i}\right)+u_{j}\left(z_{i}\right)+p\right)+\exp \left(\beta\left(u_{i}\left(z_{i}\right)+u_{j}\left(z_{j}\right)\right)\right) .\right.
\end{aligned}
$$

Note that the equality of noise parameters $\beta_{i}=\beta$ for all $i \in N$ is essential to eliminate $p$ from the numerator of (13). After trade takes place, $i$ possesses $z_{j}$ and $j$ possesses $z_{i}$ and the monetary transfer $p$ takes place from $i$ to $j$. When these agents meet again, the probability of trade (to restore the original endowments) given $-p$ is obtained by exchanging $z_{i}$ and $z_{j}$ in the above expressions when the transfer is $-p$. Namely, the probability of trade is

$$
\frac{\exp \left[\beta\left(u_{i}\left(z_{i}\right)+u_{j}\left(z_{j}\right)\right)\right]}{H(-p)}
$$

where the denominator is obtained by exchanging $z_{i}$ and $z_{j}$ in the last equality of (14). This fact is crucial for obtaining the stationary distribution in a closed form.

Note also that we have the following property: 
Lemma 9 The house allocation $z(t)$ follows a Markov chain.

This means that, despite the presence of the divisible commodity "money," we can restrict our attention to the allocation of houses, whose evolution can be described as a Markov chain on a finite state space. Intuitively, this is implied by the absence of income effects of the quasi-linear utility: the preferences over goods, and therefore the law of motion, are not affected by how much income each agent possesses. Let us now denote the Markov chain's stationary distribution by $\mu^{z}$.

Just like in the barter model with additive random utility, we have the following result.

Proposition 10 In the house allocation problem with money, the stationary distribution for the allocation of houses is given by

$$
\mu^{z}(z)=\frac{\exp \left[\beta \sum_{i \in N} u_{i}\left(z_{i}\right)\right]}{\sum_{z^{\prime} \in Z} \exp \left[\beta \sum_{i \in N} u_{i}\left(z_{i}^{\prime}\right)\right]} .
$$

Proof. Let $\operatorname{Pr}\left(z, z^{\prime}\right)$ be the transition probability from state $z$ to $z^{\prime}$. Again we will show the detailed balance condition:

$$
\mu^{z}(z) \operatorname{Pr}\left(z, z^{\prime}\right)=\mu^{z}\left(z^{\prime}\right) \operatorname{Pr}\left(z^{\prime}, z\right) .
$$

(Recall that summing both sides over $z^{\prime}$ shows that $\mu^{z}$ is the stationary distribution). To show (16), it is sufficient to prove that

$$
\exp \left[\beta \sum_{k \in N} u_{k}\left(z_{k}\right)\right] \operatorname{Pr}\left(z, z^{\prime}\right)=\exp \left[\beta \sum_{k \in N} u_{k}\left(z_{k}^{\prime}\right)\right] \operatorname{Pr}\left(z^{\prime}, z\right) .
$$

If $z^{\prime}$ cannot be obtained by a pairwise trade from $z$, then (17) is satisfied because $\operatorname{Pr}\left(z, z^{\prime}\right)=$ $\operatorname{Pr}\left(z^{\prime}, z\right)=0$. Otherwise, $z^{\prime}$ is obtained from $z$ when a pair of agents trade, in which case let us denote the pair by $(i, j)$, where $i<j$. Hence, we have

$$
\begin{aligned}
& z_{k}^{\prime}=z_{k} \text { for } k \neq i, j \text { and } \\
& z_{i}^{\prime}=z_{j} \text { and } z_{j}^{\prime}=z_{i} .
\end{aligned}
$$

Then, we have

$$
\operatorname{Pr}\left(z, z^{\prime}\right)=q(i, j) \int_{-\infty}^{\infty}\left[\frac{\exp \left[\beta\left(u_{i}\left(z_{j}\right)+u_{j}\left(z_{i}\right)\right)\right]}{H(p)}\right] f_{i j}(p) d p
$$

where $H(p)$ is given by (14). Recall that $q(i, j)$ is the probability that the pair $(i, j)$ meets, that given $p$ the exchange occurs with probability in the square bracket (see (13)), and that $p$ is proposed according to density $f_{i j}$.

Similarly, given (15) one can deduce that

$$
\operatorname{Pr}\left(z^{\prime}, z\right)=q(i, j) \int_{-\infty}^{\infty}\left[\frac{\exp \left[\beta\left(u_{i}\left(z_{i}\right)+u_{j}\left(z_{j}\right)\right)\right]}{H(-p)}\right] f_{i j}(p) d p .
$$

By the symmetry assumption on $f_{i j}(\cdot)$, i.e., $f_{i j}(p)=f_{i j}(-p)$, we have

$$
T \equiv \int_{-\infty}^{\infty} \frac{f_{i j}(p)}{H(p)} d p=\int_{-\infty}^{\infty} \frac{f_{i j}(-p)}{H(-p)} d p=\int_{-\infty}^{\infty} \frac{f_{i j}(p)}{H(-p)} d p,
$$


so that

$$
\operatorname{Pr}\left(z, z^{\prime}\right)=q(i, j) T \exp \left[\beta\left(u_{i}\left(z_{j}\right)+u_{j}\left(z_{i}\right)\right)\right]
$$

and

$$
\operatorname{Pr}\left(z^{\prime}, z\right)=q(i, j) T \exp \left[\beta\left(u_{i}\left(z_{i}\right)+u_{j}\left(z_{j}\right)\right)\right]
$$

Hence, the desired condition (17) holds since, using (18), either side of this condition is equal to

$$
q(i, j) T \exp \left[\beta\left(\sum_{k \in N} u_{k}\left(z_{k}\right)+u_{i}\left(z_{j}\right)+u_{j}\left(z_{i}\right)\right)\right] .
$$

Therefore, one obtains the same limiting results as in Corollaries 1 and 2 as $\beta \rightarrow \infty$ :

Corollary 11 In the house allocation model with money, if the noise is symmetric $\beta_{1}=\cdots=$ $\beta_{I}=\beta$ and $\beta \rightarrow \infty$, the stationary distribution places probability 1 on the goods allocation(s) that maximizes the Bentham utilitarian social welfare function $\sum_{i \in N} u_{i}\left(z_{i}\right)$.

Corollary 12 In the house allocation model with money, if the noise parameter is $\beta_{i}=\lambda_{i} \beta$ for all $i \in N$ and $\beta \rightarrow \infty$, the stationary distribution places probability 1 on the allocation(s) that maximizes the corresponding weighted utilitarian social welfare function $\sum_{i \in N} \lambda_{i} u_{i}\left(z_{i}\right)$.

The following remarks are in order:

Remark 1: The results can be extended to an exchange economy in which there are $K$ indivisible goods (apart from money) and where an agent can hold any subset of the indivisible goods. To do this, as in Section 2, one needs to assume that the proposal distribution in each meeting is uniform.

Remark 2: The results can also be extended to a process in which coalitions, not only pairs, trade. To do this, the bargaining procedure played by coalition $S$ begins with the draw of transfers $p=\left(p_{i}\right)_{i \in S}$ that is balanced, i.e., $\sum_{i \in S} p_{i}=0$. One should continue to assume that the transfer density is symmetric around 0: $f_{S}(p)=f_{S}(-p)$. Then, one can replicate the same steps in the above proof to reach identical conclusions.

\section{Related Work}

Shapley and Scarf (1974) present basic properties of a special case of discrete allocation problems we considered, known as the house allocation problem, where each agent is assigned exactly one object. Uzawa (1962) studies a deterministic barter process for divisible goods, in a setting where the trading process never gets stuck on inefficient states.

Our work generalizes a result due to Ben-Shoham, Serrano and Volij (2004). In that paper, only pairwise trade in the house allocation problem without money was considered, and the persistent shocks are "mistakes" in decision-making. ${ }^{5}$ In particular, they assume that, when an agent has

\footnotetext{
${ }^{5}$ In another paper, Serrano and Volij (2003) explore coalitional trade. Their exchange process is quite different though, because it gives a special role to agents' initial endowments. This leads to connections with the Walrasian and core allocations.
} 
his $k$ th best house, the probability of accepting her $m$ th best house $(m>k)$ has the order of $\varepsilon^{m-k}$, where $\varepsilon \in(0,1)$ is a small number. This is a particular formulation of mistake probabilities where more serious mistakes are less likely. They show that when the randomness is vanishingly small (as $\varepsilon \rightarrow 0$ ), the allocation that minimizes envy is selected in the long run. Agent $i$ 's envy level is the number of people who have better houses than agent $i$ (according to $i$ 's preferences). The envy in the society is the sum of individual agents' envy levels. The current paper shows that there is a more general mechanism at work behind this result. First, we note that their specification of noise can be related to the logit model. Let $N$ be the number of houses/agents and let us assume that agent $i$ 's utility for her $k$ th best house $x_{i}$ is $u_{i}\left(x_{i}\right)=N-k+1$ (so that the utilities of the $N$ houses are $1,2, \ldots, N$, where $N$ is the utility of the best house). A straightforward calculation shows that we obtain their specification of mistake probabilities, when we add the logit noise term to this utility function. Second, one can see that the envy is equal to $\sum_{i}\left(N-u\left(x_{i}\right)\right)$, and minimizing this is equivalent to maximizing the utilitarian social welfare $\sum_{i} u\left(x_{i}\right)$. We have found that the driving force of their result is that the logit noise model maximizes the utilitarian social welfare (and this is true for any specifications of utility functions). Furthermore, we are able to derive the stationary distribution not only when the noise is negligible but also when the randomness is large. This addresses the concern that it takes a very long time to see the predictions of stochastic evolutionary models.

Several papers have used logit noise in dynamic adjustment processes (see Durlauf (1997) and the references therein). The most closely related work to ours is Blume (1997), who obtained a closed form expression of the stationary distribution for any level of noise when the following three conditions are satisfied: ${ }^{6}$ (i) players play a potential game (i.e., each player's best reply function is the same as in a game in which players have an identical payoff ("potential")), (ii) at each moment of time, only one player can adjust, and (iii) all players have an identical precision parameter for their logit random utility terms. Blume (1997) shows that the stationary distribution is given by

$$
\frac{\exp [\beta P(a)]}{\sum_{a^{\prime} \in A} \exp \left[\beta P\left(a^{\prime}\right)\right]},
$$

where $P$ is the potential, $A$ is the set of strategy profiles, and $\beta$ is the common precision parameter measuring the level of utility noise. Young and Burke (2001) present an intriguing application of Blume's result to explain the distribution of agricultural contracts in Illinois. Our models are different from Blume's in that they do not satisfy the above conditions. The technical contribution of our paper is to show that a similar closed form expression can be obtained for a wider class of situations where those conditions are not necessarily satisfied.

\section{References}

Aarts, E. and J. Korst (1989), Simulated Annealing and Boltzmann Machines, Chichester: John Wiley \& Sons.

Ben-Shoham, A., R. Serrano and O. Volij (2004), "The Evolution of Exchange," Journal of Economic Theory 114, 310-328.

\footnotetext{
${ }^{6}$ See also Young and Burke (2001) and Sandholm (2003).
} 
Blume, L. (1997), "Population Games," in B. Arthur, S. Durlauf, and D. Lane (eds.), The Economic System as an Evolving Complex System II, Reading, MA:Addison-Wesley.

Durlauf, S. N. (1997), "Statistical Mechanics Approaches to Socioeconomic Behavior," in B. Arthur, S. Durlauf, and D. Lane (eds.), The Economic System as an Evolving Complex System II, Reading, MA:Addison-Wesley.

Harsanyi, J. and R. Selten (1972), "A Generalized Nash Solution for Two-Person Bargaining Games with Incomplete Information. Part II," Management Science 18, 80-106.

Kalai, E. (1977), "Nonsymmetric Nash Solutions and Replications of Two-Person Bargaining", International Journal of Game Theory 6, 129-133.

Kandori, M., G. Mailath, and R. Rob (1993), "Learning, Mutations and Long Run Equilibria in Games," Econometrica 61, 29-56.

Kaneko, M. (1980), "An Extension of the Nash Bargaining Problem and the Nash Social Welfare Function," Theory and Decision 12, 135-148.

Roth, A. (1979), Axiomatic Models of Bargaining, Lecture Notes in Economics and Mathematical Systems No. 170, Springer Verlag.

Sandholm, W. H. (2003), "Pigouvian Pricing and Stochastic Evolutionary Implementation," http://www.ssc.wisc.edu/ whs

Serrano, R. and O. Volij (2003), "Mistakes in Cooperation: the Stochastic Stability of Edgeworth's Recontracting," Working Paper 2003-23, Department of Economics, Brown University.

Shapley, L. and H. Scarf (1974), "On Cores and Indivisibility," Journal of Mathematical Economics 1, 23-28.

Uzawa, H. (1962), "On the Stability of Edgeworth's Barter Process," International Economic Review, 3, 218-232.

Young, H. P. (1993a), "The Evolution of Conventions," Econometrica 61, 57-84.

Young, H. P. (1993b), "An Evolutionary Model of Bargaining," Journal of Economic Theory 59, 145-168.

Young, H. P. and M. Burke (2001), "Competition and Custom in Economic Contracts: A Case Study of Illinois Agriculture," American Economic Review 91, 559-573. 